\title{
Functional analysis and the species design
}

\author{
Karen Neander ${ }^{1}$
}

Received: 14 May 2014 / Accepted: 29 September 2015 / Published online: 29 October 2015

(C) The Author(s) 2015. This article is published with open access at Springerlink.com

\begin{abstract}
This paper argues that a minimal notion of function and a notion of normalproper function are used in explaining how bodies and brains operate. Neither is Cummins' (1975) notion, as originally defined, and yet his is often taken to be the clearly relevant notion for such an explanatory context. This paper also explains how adverting to normal-proper functions, even if these are selected functions, can play a significant scientific role in the operational explanations of complex systems that physiologists and neurophysiologists provide, despite a lack of relevant causal efficacy on the part of such functions.
\end{abstract}

Keywords Function · Functional analysis · Mechanism · Explanation · Complexity · Organized complexity $\cdot$ Causal efficacy $\cdot$ Idealization $\cdot$ Physiology $\cdot$ Neurophysiology

What role do function ascriptions play in the operational explanations of bodies and brains that are provided by physiologists and neurophysiologists, and what notion (or notions) of function are involved?

\section{How-questions and Why-questions}

To identify the explanations that are of interest here, Mayr's (1961) distinction between How-questions and Why-questions is a good place to begin. This present paper concerns How-questions. A How-question asks how a system operates. How does a visual system enable vision? How are sleep-wake cycles controlled? How does a circulatory system circulate blood? In contrast, Why-questions ask about the origin, presence or

$\triangle$ Karen Neander

kneander@duke.edu

1 Duke University, Durham, NC, USA 
persistence of something. Why are there eye saccades? Why do we have pineal glands? Why do veins have valves? ${ }^{1}$

Mayr also drew a distinction between two main branches of biology that he called 'evolutionary' and 'functional'. ${ }^{2}$ The evolutionary biologist is concerned with Whyquestions, he says, whereas his functional biologist "is vitally concerned with the operation and interaction of structural elements, from molecules up to organs and whole individuals. His ever-repeated question is 'How?' How does something operate, how does it function?"' (Mayr 1961, p. 11). As Mayr tells us, the functional biologist answers such a question by conceptually decomposing a system into its components and these into their sub-components at multiple levels of analysis, and by describing how their diverse activities interact to produce the activities of the system as a whole.

Mayr's functional biologists are those whom I here call 'physiologists' (and 'neurophysiologists'). Mayr describes these biologists as primarily experimentalists, who isolate the specific components to be studied as much as possible and then study their operation in a few individuals. My interest here, however, is not in these individual experiments so much as in the multi-level componential analyses (i.e., functional analyses or mechanistic explanations) of complex organic systems to which the experimental results collectively contribute.

\section{The alleged division of explanatory labor}

One view that has considerable currency is that Cummins' notion of function is used in these explanations and that a (roughly) Wright-style notion or (more specifically) a notion of a selected function is not.

Wright's $(1973,1976)$ seminal idea was that the function of an entity is what it does that explains why it is there or why it has the form that it has. Wright claimed that ascriptions of such functions answer Why-questions in biology as well as in ordinary discourse. Later versions of what are known as etiological theories can differ from Wright's in significant ways. ${ }^{3}$ For instance, some tie functions explicitly to past selection. The core idea of these theories is that the function of an item is to do what it was selected to do, or what items of the type were selected to do (depending on what type of selection is involved). To simplify, I here assume that selected functions are grounded in phylogenetic natural selection (although other kinds of selection might ground selected functions too). ${ }^{4}$

\footnotetext{
1 'How-questions' and 'Why-questions' are mnemonic tags for questions that are often but are not invariably asked by using the words 'how' and 'why'.

2 Did Mayr think that only answers to How-questions were functional explanations? He might have shared Bock and von Wahlert's (1965) view that, while 'function' is ambiguous, we ought to revise in favor of using it in only one of its senses in order to avoid confusion.

3 See esp. Millikan (1989a) and also Neander (1991a), which follows Neander (1983).

${ }^{4}$ Roughly, in the phylogenetic case, the relevant selection is relatively recent and includes maintenance selection, but see Neander and Rosenberg (2012) for details on how traits are typed for this purpose and for a discussion of how vestigial traits are handled. Other forms of selection that might ground functions might include intentional selection, cultural selection, antibody selection and neural selection. Whether the etiological theory of functions can allow all such forms of 'selection' to ground functions without introducing problems cannot be taken for granted. For discussion relating to neural selection, see Garson (2012).
} 
What are often called 'causal role functions' or 'systemic functions' are described by a systemic capacity theory, the classic version of which is from Robert Cummins. ${ }^{5}$ On Cummins' original account, a function of a component of a system is its contribution to whatever complexly achieved $\left(Z^{*}\right)$ capacity of the system happens to be under analysis. Cummins tells us that a component $X$ of a system $S$ "functions as a $Z$ in $S$ (or: the function of $\mathrm{X}$ in $\mathrm{S}$ is to $\mathrm{Z}$ ) relative to an analytical account $\mathrm{A}$ of $\mathrm{S}$ 's capacity to $Z^{*}$ just in case $\mathrm{X}$ is capable of $\mathrm{Z}$-ing in $\mathrm{S}$ and $\mathrm{A}$ appropriately and adequately accounts for S's capacity to $Z^{*}$ by, in part, appealing to the capacity of $X$ to $Z$ in $S$ ” (1975, p. 762). ${ }^{6}$

We need to distinguish among three theses for which Cummins argues. Thesis 1 is that Wright-style function ascriptions do not give scientifically respectable answers to Why-questions in contemporary biology. This thesis is not my present concern, and for the most part I set it aside. But, just for a moment, let's glance at Thesis 1 . About Thesis 1 , Cummins seems to be wrong. If the human pineal gland has the selected function to secrete melatonin, then (trivially) human pineal glands were selected for secreting melatonin. So, citing the pineal glands' selected function would at least contribute to explaining why pineal glands persisted in our population. ${ }^{7}$

Thesis 2 is that function ascriptions of some sort answer How-questions. Most everyone now agrees with this, and it should be considered uncontroversial (which is not to say that it did not need to be said, when Cummins said it). ${ }^{8}$

Thesis 3 is in two parts. Thesis $3 \mathrm{a}$ is that what I shall here call 'Cummins functions' (i.e., functions as analyzed by Cummins's original analysis) are ascribed in multi-level componential analyses of how complex systems operate. Thesis $3 \mathrm{~b}$ is that Wright-style functions are not ascribed in these analyses of how complex systems operate. In my view, Cummins is right about Thesis 2 but wrong about Thesis 3 .

So, how could Wright-style functions possibly answer How-questions? Past phylogenetic selection may be a background structuring cause, to employ Dretske's (1988) term, but past phylogenetic selection is not a triggering cause in the current operation of a living system. Far-flung causal chains can explain the persistence and distribution of a component in a species. And selected function ascriptions can (in my view) in part answer Why-questions by pointing to aspects of these far-flung histories. But Thesis 3 concerns attempts to explain how organic systems operate in the here and now.

Whether a woman sleeps or lies awake tossing and turning can depend on whether her pineal gland is secreting melatonin now. But the fact that ancestral pineal glands

\footnotetext{
5 See also Hardcastle (1999) and Craver (2001).

6 Cummins' symbols are changed to conform to those used elsewhere in this paper.

7 Wright (1976) and Neander (1991b) discuss the relation between functional explanations and teleological explanations. Note that Thesis 1 raises the question of whether natural selection explains why individuals have the adaptations they do. See Sober $(1994,1995)$ and Neander (1991b, 1995) for initial discussion. It is important to separate metaphysical issues, to do with an individual's historical essence, from methodological issues to do with explaining adaptations, and in that sense answering Paley's question, as subsequent discussion has made apparent. In any event, these issues are not relevant to the main line of argument in this paper.

${ }^{8}$ Hempel (1965, Chap. 12), like Wright (1976), viewed function ascriptions as explaining the presence of the item with the function. Cummins was not the first to see that Thesis 2 was true, but his paper encouraged a shift in thinking among philosophers on the nature of functional explanations.
} 
were selected for secreting melatonin in the past will not send sweet slumber her way. So I willingly grant that a system that lacked a selection history and yet was a narrow - current, intrinsic — physical duplicate of a system that had one (the so-called 'Swamp creature') would operate in the same way in the same situation (ignoring, for simplicity, the indeterminacy of the fundamental laws of physics). ${ }^{9}$

Even some of the main proponents of the etiological theories have sometimes accepted Thesis 3, seemingly for the reasons just given. Godfrey-Smith (1993, p. 189) comments with approval that there are "entire realms of functional discourse, in fields such as biochemistry, developmental biology and much of the neurosciences... in which the attractive account of functions has always been that of Cummins (1975)." Godfrey-Smith believes that Wright-style functions and Cummins-style functions both play explanatory roles but in different types of explanation. He emphasizes that Cummins functions are needed to explain how systems operate because there is nothing special about causal contributions qua causal contributions for which there was selection. He says that he follows Ruth Millikan in this. ${ }^{10}$

And Millikan (1989b), having elucidated how selected functions answer Whyquestions, adds that, "with the Cummins sense of function goes another sense of "functional explanation"” (p. 175). She goes on to explain that Cummins-style functions are ascribed in multi-level componential analyses of complex capacities, which answer How-questions.

Griffiths (2006, p. 3) also says that "unless anatomy, physiology, molecular biology, developmental biology, and so forth turn their attention to specifically evolutionary questions, they investigate function in the causal [role] sense."11

Enough has been said along these lines for this bifurcation in explanatory roles to be now routinely presented as if it were enshrined in the etiological and systemic capacity theories. For example, Davies (2001, p. 28) says, "The theories appear to have distinct explanatory aims. While selected functions explain the persistence and proliferation of a trait in a population, systemic functions explain how a system exercises some capacity." And again, "advocates of selected functions explain, for example, why the mammalian heart persisted, while advocates of systemic functions explain how the circulatory system exercises the capacity of circulating nutrients..." (Davies 2001, p. 41). ${ }^{12}$ Davies demurs on the division of explanatory labor but only to dispute (as Cummins did) whether selected functions are needed at all. Along the same lines, Huneman (2013,p. 2) says that supporters of both the etiological and systemic capacity theory "Both acknowledge that 'function' is a concept used in some explanations, but they diverge from the first step because the etiological account thinks that the function

\footnotetext{
9 'Swampman' is the name given by Davidson (1987) to his (imaginary) molecule-for-molecule doppelganger, who has no history beyond the separate histories of each its physical elements. Davidson raises Swampman in relation to intentionality. Boorse (1977) raises a similar example in relation to functions. See Neander (1991a, 1996) and other papers in the 1996 volume for discussion.

10 Millikan (2002), as well as Neander (1991b), endorses a view like the one developed in more detail here. See footnote 27.

11 Bouchard (2013) also seems to take this division of explanatory labor for granted when he says of Amundson and Lauder (1994) that they "use examples from physiology and other biological fields that cannot be said to use historical functional concepts."

12 To be fair, Davies (2001, p. 41) says that the etiological theory is typically cast in these terms.
} 
of $\mathrm{X}$ being $\mathrm{Y}$ explains the presence of $\mathrm{X}$ whereas, for the causal role theorist, the function of $\mathrm{X}$ being $\mathrm{Y}$ explains or contributes to an explanation of the general proper activity of a system which includes X."

I could pile on more and more quotations along these lines, but this is enough to make the point - namely, that one could easily gain the impression that everyone now agrees with Cummins that the physiologists' How-questions are answered by Cummins functions and not Wright-style selected functions. But the issue needs to be reconsidered. First off, Thesis $3 \mathrm{~b}$ is not integral to the etiological theory of functions. The etiological theory of functions provides an analysis of a certain notion of function. It does not try to settle the explanatory role of the notion so analyzed. I ask readers to consider it an open question for now whether selected function ascriptions play a role in answering the physiologists' How-questions. Huneman's reference to the 'general proper' activity of a system hints at one reason to suspect that they do.

\section{Minimal and normal-proper functions}

In my view, answers to How-questions in physiology clearly advert to what I will here call 'minimal functions' and 'normal-proper functions'. Neither of these are Cummins functions (remember that Cummins functions are functions as originally defined by Cummins). This section first elucidates how 'minimal function' and 'normal-proper function' are defined for the purposes of this paper. Then it reviews the reasons why Cummins functions are neither minimal nor normal-proper, and why selected functions are at least prima facie plausible candidates for identification with normal-proper functions.

A minimal function of an entity is just something that it does. In some contexts, it is usual to speak of the mere activity of an entity as its functioning. In this sense of 'function', we speak of a system's actual functioning even if it is pathological. Minimal functions are mere doings, mere activities, which can contribute to outcomes that we might or might not be interested in explaining. ${ }^{13}$

A notion of a normal-proper function, in contrast, underwrites talk of normal function, of systems functioning properly, of malfunction, dysfunction, abnormal functioning, impaired functioning and functional deficits. The notion of normal-proper function is here identified by ostension as the notion that most centrally underwrites this kind of talk in biology. ${ }^{14}$ This notion of function often underwrites talk of an entity having a function. Note that this ostensive definition presupposes no particular analysis of this notion of normal-proper function. It does not presuppose an etiological analysis of it; it leaves the question open as to whether an etiological analysis is the best analysis of it.

A few points about the notion of normal-proper function are (rightly) generally accepted and worth remembering. First, the normal-proper function of an entity is not a mere effect, activity or disposition to engage in an activity. The heart has the normal-

\footnotetext{
13 This is similar to Bock and von Wahlert's (1965) notion of function as activity.

14 I assume that there is a central malfunction-permitting notion of function involved. Kraemer (2014), for example, argues that there is more than one although he does not argue that more than one is central.
} 
proper function to pump blood but not to make whooshing noises, even though hearts do both (and even though whooshing noises are useful diagnostic aids). Wright (1973, 1976) emphasized the difference between claiming that an item has the function to $\mathrm{Z}$ versus claiming that it serves the function to $\mathrm{Z}$. The soldier's belt buckle does not have the function to stop a bullet, he said, but if it accidentally stops a bullet then it serves that function. Similarly, hearts can act as diagnostic aids, even if they do not have the function of assisting physicians in diagnosing cardiac problems. This distinction is sometimes called 'the accident-function distinction'. Normal-proper functions respect the accident-function distinction.

Nor need a (token) component perform its normal-proper function. The appropriate occasion might never arise (an antelope born in a zoo might never need to use its long legs to escape from predators). Or the environment might not cooperate (a diver's lungs will not absorb oxygen if she is deep-sea diving when her tank runs out). Also, in this sense of 'function', a token trait can malfunction. ${ }^{15}$ It might lack the ability to perform its normal-proper function. A person's pancreas can have the function to produce insulin even if it is unable to produce insulin. And there is no incoherence in the idea that functional impairment could become typical in a population for a time, in a pandemic or due to an environmental disaster. The relevant function-dysfunction distinction does not seem to be simply the typical-atypical or expected-unexpected activity distinction. This much is fairly uncontroversial. ${ }^{16}$

Most will agree that selected functions are, at least at first blush, plausible candidates for identification with normal-proper functions. Let's quickly run through the points made above concerning normal-proper function, but now with selected functions in mind. The heart does not have the selected function to make whooshing noises. Specific tokens need not perform their selected functions because the appropriate occasion might not now arise or the environment might not now cooperate. And there is the possibility of malfunction because the selected functions of present tokens depend, not on the current dispositions of individuals but on past selection operating on ancestral traits. For the same reason, such malfunction could become typical in the present population.

There is talk of functional 'norms' and of the associated notion of function as 'normative'. These words can create a great deal of misunderstanding. On the etiological theory, functional 'norms' (so to speak) are purely descriptive. On the etiological theory, a 'normative' notion of function is one that permits the possibility of malfunction and is sensitive to the function-dysfunction and accident-function distinction. It

\footnotetext{
15 Boorse (2002) (contra Boorse 1977) claims that a token trait does not have a (normal-proper) function that it cannot perform. However, he does not deny the possibility of malfunction and so his preferred way of speaking is a terminological variant for present purposes. I say that a malfunctioning token loses or lacks the ability to perform its function (with normal efficiency). He says that it loses or lacks the ability to perform (with normal efficiency) not its function but a normal function of traits of the type. Either way, dispositions and indispositions divide into two groups. There are dispositions that components can lack without malfunction and dispositions that components cannot lack without malfunction. Lungs that lack the ability to produce gametes and digest cellulose are not malfunctioning because these are not the normalproper functions of lungs. In contrast, if lungs lack the ability to absorb oxygen then they are malfunctioning because this is a normal-proper function of lungs.

16 Boorse (1977, 2002) argues that the notion of normal function(ing) is statistical but not simply statistical. On this, see the penultimate section of this paper and footnote 37 .
} 
draws these distinctions in terms of what entities were selected to do as opposed to what they are disposed to do. However, to repeat, the ostensive definition for "normalproper function' does not presuppose the identification of normal-proper function with selected function.

Most will also agree that, at least at first blush and as originally defined, Cummins functions are problematic as candidates for identification with normal-proper functions. This is old ground, not to be rehashed at length here, but I want to briefly remind readers of this old ground and convey some sense of it to those not familiar with it. ${ }^{17}$

First off, Cummins' notion seems to be insensitive to the function-dysfunction distinction. He says, for example, "If the function of something in a system $\mathrm{S}$ is to pump, then it must be capable of pumping in $S$ " (1975, p. 757). In contrast, the possibility of malfunction requires that ' $\mathrm{X}$ has the function to $\mathrm{Z}$ ' be compatible with ' $\mathrm{X}$ lacks the capacity to Z'. If we read Cummins' statement as talking of types (such as canine hearts) rather than tokens (such as Fido's heart) then his account does not clearly preclude malfunction; however, it is at best undeveloped in this respect (more on this later). ${ }^{18}$ Further, on Cummins' account, the complex $\left(\mathrm{Z}^{*}\right)$ capacity to which a component with a function must contribute is determined by a researcher's explanatory aims and, since physiologists are interested in explaining pathological processes, such as the growth of a tumor, a component can have a Cummins function by virtue of its causal contribution to an organisms' malignancy. So in that respect too, Cummins functions are-as originally analyzed by Cummins-insensitive to the standard function-dysfunction distinction.

Nor is Cummins' notion sensitive to the standard accident-function distinction. ${ }^{19}$ His original account did not attempt to capture this distinction. Indeed, in his analysis (cited in the previous section) Cummins equates - Wright would say 'conflates' - ' $\mathrm{X}$ functions as $a \mathrm{Z}$ in $\mathrm{S}$ ' with 'the function of $\mathrm{X}$ in $\mathrm{S}$ is to $\mathrm{Z}$ '. ${ }^{20}$ Yet, if we observe the standard accident-function distinction, the belt buckle can function as a bullet stopper without having the function to stop bullets, and the heart can function as a diagnostic aid without having the function to help with diagnosis.

Cummins functions are not the same as minimal functions either. Minimal functions are mere doings, activities or causal contributions to outcomes, whether or not these outcomes are complexly achieved and whether or not anyone wants to explain them. ${ }^{21}$

In contrast, Cummins functions are mind-dependent in two main ways. Researcher interests determine the complex $\left(Z^{*}\right)$ capacity of a system to which a Cummins function contributes, and the pragmatic features of the explanatory context determine Cummins functions in other ways. For example, they determine the boundaries of the system

\footnotetext{
17 Neander (1991a, p. 81) makes several of the following points.

18 Cummins and Roth (2010, p. 78) opt for an explicitly instrumentalist treatment of functional norms in a footnote that asks, rhetorically, how else we can make sense of functional norms, "short of saying that normativity is built into the very fabric of reality." Of course, proponents of the etiological theory argue that functional norms are descriptive, not prescriptive.

19 As Millikan (1989b) argues.

20 See Cummins (1975, p. 762).

21 I take it that a component $\mathrm{X}$ can, by Z-ing, causally contribute to something $\mathrm{Z}^{*}$-ing, whether or not anyone wants to explain how $Z^{*}$ occurs. Of course, this is not entirely uncontroversial.
} 
under analysis. ${ }^{22}$ Consider, to return to artifacts for a moment, how Cummins' account applies to them. Simple artifacts, such as spoons, cups and belt buckles, are not complex systems. But Cummins can allow that spoons and the like have functions by allowing them to count as parts of systems that include their users. The spoon, for example, can be ascribed the function of carrying food to the mouth if someone is interested in explaining how a person is nourished and so draws the boundaries of the system widely enough to include the spoon.

This pragmatic approach to delineating systems must be kept in mind when we assess the theory. For example, it bears on whether Cummins' account respects the accident-function distinction. If the soldier's belt buckle is counted as part of a complex system that includes the soldier (as it must, if it has the function of holding up the soldier's trousers), it can also be ascribed the function of stopping a bullet, relative to an explanation of the soldier's ability to survive. Plus, a human heart can be counted as part of a complex system that includes a doctor and a stethoscope and be ascribed the function of making whooshing noises relative to an explanation of how doctors diagnose heart problems.

In sum, as originally analyzed, Cummins functions do not respect the standard function-dysfunction or accident-function distinctions. Cummins (1975) made no attempt to capture these distinctions. Of course, similar accounts have been further developed in the decades since (more on this later). The material in this section is not an argument against the claim that modified Cummins-style functions are the biologists' normal-proper functions. The point for now is only that Cummins functions, as originally analyzed by Cummins, are neither normal-proper functions nor minimal functions. This must be taken into account when considering Thesis 3 .

\section{Questioning Cummins' Thesis 3}

Recall that Thesis 3 says that physiologists speak of Cummins functions and not Wright-style selected functions in explaining how complex living systems operate. In my view, physiologists speak of minimal functions and normal-proper functions in this context and, as we just saw, neither are Cummins functions. This section discusses some preliminary thoughts in favor of taking this counter claim seriously.

To be clear, I completely agree with Cummins (and Mayr among others) that physiologists give multi-level componential analyses of how bodies and brains operate. And of course I agree that physiologists must advert to the diverse causal contributions of components to the complexly achieved capacities of these systems in doing so. In my view, this should be considered uncontroversial, details aside. But it would be a colossal mistake to think that it obviously follows that Cummins functions are cited in such explanations. It would be a colossal mistake to think that Thesis 3 a obviously follows.

Causal contributions qua causal contributions are one thing. Causal contributions qua contributions to complexly achieved capacities of pragmatically delineated sys-

\footnotetext{
22 Though, as an anonymous referee points out, Davies (2001), who is sympathetic to an analysis of functions broadly in the same spirit as Cummins', argues for a mind-independent delineation of a system.
} 
tems that someone wants to explain are another. The former are minimal functions, whereas the latter are Cummins functions. I suspect that some of the philosophers who express support for Thesis 3 a are really in favor of the view that minimal functions play the relevant explanatory role and are overlooking the distinction.

There are explanatory aims when anyone tries to explain complex or, for that matter, simple capacities. And which causal contributions ought to be mentioned in a given explanatory context will depend on one's aims. But, on Cummins' account, if there are no relevant explanatory aims, then there are no functions. Explanatory aims are constitutive for Cummins functions. Explanatory aims are not constitutive for minimal functions. Cummins functions supervene on the mental states of people seeking explanations, whereas minimal functions do not.

Some might feel impatient with this distinction, but it is far from a distinction without a difference. In fact, the naturalization program in philosophy of mind might hang on it for it makes all of the difference in the world as to whether mental phenomena can be explained in terms of non-mental phenomena by adverting to functions. It is a problem for functionalist theories of mind if the relevant functions are Cummins functions. And it is a problem for teleosemantic theories if the relevant normal-proper functions are, or are constitutively tied to, Cummins functions. ${ }^{23}$ The needs of philosophy of mind do not trump those of physiology or neurophysiology, but this is no distinction over which to shrug shoulders.

Also, while Thesis $3 \mathrm{~b}$ denies that physiologists advert to Wright-style functions, physiologists speak of normal-proper functions of some kind. Those function pluralists who are also proponents of the etiological theory of normal-proper function should be especially wary of accepting Thesis $3 \mathrm{~b}$.

In case readers doubt that physiologists and neurophysiologists speak of normalproper function in their serious journal articles, let me add that a quick perusal of the titles and abstracts of papers in the American Journal of Physiology, for example, would soon make the prevalence of talk of normal-proper functions clear. Reference to disease, dysfunction, abnormal, impaired or pathological functioning is commonplace in titles and abstracts. ${ }^{24}$ It also occurs, frequently, in the papers. For example, in a paper with the tempting title, "Tissue-specific downregulation of dimethylarginine dimethylaminohydrolase in hyperhomocysteinemia," the word 'dysfunction' alone is used 22 times. $^{25}$

Many areas of science give multi-level componential analyses without ascribing functions, other than in a minimal sense. The formation of a planetary system is a

\footnotetext{
23 For an introduction to functionalist theories of mind, see Rey (1997) or Kim (2010) and for a survey of teleosemantic theories of mental content, see Neander (2011).

24 For example, in the August 2008 issue of the American Journal of Physiology, the first two titles and an excerpt of the abstracts are as follows. Title 1: Abnormalities of IGF-I signaling in the pathogenesis of diseases of the bone, brain, and fetoplacental unit in humans. Abstract 1 (excerpt): This article will review current information on IGF-I signaling and illustrate recent results demonstrating how impaired IGF-I signaling and action may contribute to the pathogenesis of human diseases. Title 2: Neural control of the anorexia-cachexia syndrome. Abstract 2 (excerpt): [D]isease-induced changes in fatty acid oxidation within hypothalamic neurons may contribute to the dysfunction of the hypothalamic melanocortin system. These excerpts are taken from Laviola et al. (2008) and Laviano et al. (2008), respectively.

25 Dayal (2008).
} 
complex process that theorists explain at multiple levels of analysis, from stars to sub-atomic particles. Yet, contemporary cosmologists do not claim that stars have the function to send heavy elements into the interstellar medium to help form vast clouds of molecular matter, that pre-planetary clumps of matter have the function to collide and accrete into larger clumps, or that the different elements and compounds have functions to behave in the ways in which they must if a solar system like ours is to form. Certainly, contemporary cosmologists do not ascribe malfunction-permitting functions to components in explaining the formation of solar systems. ${ }^{26}$

To recap, Thesis 3 a claims that Cummins functions are the functions needed to explain how bodies and brains operate. This is not motivated by the claim that what explains how bodies and brains operate are the causal contributions of their components. Thesis $3 b$ denies that selected functions have a role in explaining how systems operate. As mentioned before, the etiological theory is often routinely presented as if it denies that selected functions have a role in explaining how systems operate, but the etiological theory makes no such denial. Some proponents of it make such a denial, but they might be mistaken, for some notion of normal-proper function has some role in physiology and neurophysiology.

\section{Normal-proper function and the generalization problem}

At this juncture, someone might reply that, for all I have said so far, it is unobvious that physiologists and neurophysiologists use a notion of a normal-proper function for any significant scientific purpose in explaining how complex organic systems operate. Fair enough, but more is to come. This section considers its role without assuming any particular analysis of normal-proper functions. The next considers its role in more detail, on the assumption that normal-proper functions are selected functions.

It must be allowed that the selected functions of a system's components will lack causal efficacy with respect to the system's current operation. This was mentioned earlier as a reason to doubt that selected functions explain how complex systems operate. But now note that normal-proper functions must lack relevant causal efficacy no matter how they are analyzed. So, if lack of causal efficacy were a reason to doubt that selected functions explain how complex systems operate, lack of relevant causal efficacy will also be a reason to doubt that normal-proper functions (no matter how they are analyzed) can play such a role. There is always a possible gap-given the possibility of malfunction-between what a component does and what it would do if it were functioning properly. And it is what components $d o$ - minimal functions - that produces physiological outcomes. If a woman is gasping for breath because her lungs are malfunctioning, the fact that her lungs have the normal-proper function to absorb oxygen will not save her. Normal-proper functions will lack relevant causal efficacy whether or not they are selected functions. They will lack relevant causal efficacy

\footnotetext{
26 The cosmologists' and the physiologists' explanations are both mechanistic explanations, in the sense elucidated by Craver and Darden (2013). Functional explanations are a special kind of mechanistic explanation.
} 
even if they are a special subset of Cummins functions or are modified Cummins-style functions, for instance.

Causal claims may take the form of counterfactuals. Even so, neither having a normal-proper function nor having a selected function (whether or not these are the same) is a causally efficacious property of a component. To say that a malfunctioning component would do otherwise in the same context if it were functioning properly or if it were able to do what components of the type were selected to do is to say that it would do otherwise if it had different causal powers from those that it has.

So why do physiologists and neurophysiologists speak of normal-proper function? Mayr (1992, p. 131) says that, "one physiological discovery after another resulted from asking and answering Why-questions." To borrow his example, Harvey discovered that hearts circulate blood after wondering why veins have valves. This role in discovery-suggesting answers to How-questions by answering Why-questions-is one role that ascriptions of normal-proper function (especially if normal-proper function is construed as selected function) can play in physiology even if such ascriptions only directly answer Why-questions. But can citing normal-proper function play a more direct role in explaining how bodies and brains operate, despite a lack of relevant causal efficacy on the part of such functions?

Physiologists and neurophysiologists, like many other scientists, are in the business of discovering "the mechanisms, often hidden, that produce the phenomena that we want to understand," as Salmon (1993, p. 89) puts it. Nevertheless, I dispute the stronger claim that is sometimes added, to the effect that only causally efficacious properties should be invoked in such explanations. Kim (2000, p. 31) affirms this stronger claim, for instance, when he says that, "for any phenomena to have an explanatory role, its presence or absence in a given situation must make a difference-a causal difference." People can mean different things by 'explanatory role', and so let me put it this way. My claim is that citing the normal-proper functions of the components of a system can play a significant scientific role in the answers to How-questions that physiologists and neurophysiologists provide, despite the fact that the having of a normal-proper function is not a causally efficacious property of a component.

What is this role? In part, the answer is that physiologists solve their generalization problem by speaking of normal-proper functions. All branches of science face generalization problems; however, the physiologists' problem is extreme, due to the immense complexity of living systems and the enormous variation that results. Describing the operation of an immensely complex system, such as a human body or brain, or even a single neuron, is a huge challenge in itself. But the further problem is that the greater the complexity, the more variables there are that can vary from one instance to the next, or in a single instance over time. The more variables there are, the more potential there is for variation among different individuals, or even in a single individual over time as it develops, matures, becomes sick or injured, recovers and so on. It is in the nature of living things that a vast deal of potential variation is realized. Complex living systems tend to have sui generis genomes and many fluid as well as some more rigid interactions with environmental features during development and beyond. There is a tremendous amount of variation in a single species, as is the case with the six billion or so humans alive today. Yet, physiologists and neurophysiologists collectively contribute to useful, general descriptions of how types of bodies and brains operate. 
They do so by describing systems that function normally or properly. Ironically, it is the uniqueness of actual individual functioning that accounts for why physiology is so steeped in talk of normal-proper function.

\section{The system that functions as designed}

Of course, other sciences can also make generalizations, by using statistics. But since bodies and brains are, in part, the product of selection, a special solution to the generalization problem is available to the physiologists, which is unavailable to the geologists and cosmologists. In this section, I describe in more detail what I take to be the physiologists' solution to their generalization problem, on the assumption that the normal-proper functions are selected functions. There has been prior recognition of this possibility. ${ }^{27}$ But it has been largely ignored and anyway needs to be more fully developed.

On the etiological theory, a normal system is one in which each component that was selected to do something has the ability to do what it was selected to do. On the etiological theory, a normal system is one that is disposed to function 'as designed', so to speak. Some (maybe much) of what I say here will be true on other understandings of normal-proper function too. But, to elucidate the physiologists' strategy in more detail, I need to assume my understanding of the relevant notion of function. (Who else's?) I leave it to readers to decide what will hold true on their understanding of the relevant notion of function if it differs from mine. So this section describes what I take to be the theoretical purpose served by ascriptions of normal-proper function on the assumption (not to argue) that the etiological theory is true. ${ }^{28}$

How might describing a complex of properly functioning adaptations contribute to a useful, as well as a general description of how living systems operate? I will enumerate the ways, but the points are interrelated and not all clearly distinct.

First is the fact that, while heritable variation is the raw material on which selection works, selection has pushed many adaptive traits to fixation and so towards something that approximates a single species design. The description of a normal system, as a system in which components that were selected to do something can do what they were selected to do, describes the species design to the extent that it exists.

\footnotetext{
27 Neander (1991b, p. 467) says that, "the physiologist's analysis of the human digestive or circulatory system, for example, proceeds by way of a decomposition of the system into its functionally individuated parts ... And then a description of the proper function of each part is given, that being the contribution of each part to the functioning of the whole, when the system is functioning properly." Millikan (2002, p. 52) expresses a similar view when she says, "Cummins emphasized the project of finding out how the biological system works, not just finding out what it does. But, of course, finding out what it does in detail, what all its proper functions are and all the proper functions of all its parts, IS finding out how it works." Brandon (2013) says that a causal-role functional analysis of how a system is adaptive in its current environment as well as an analysis of the way in which a system is composed of adaptations can be of value. Garson (2013) provides a useful discussion of mechanism that also complements this paper. (Early circulated drafts and conference presentations of this paper precede the papers by Garson and Brandon.)

28 Of course, it speaks in favor of an etiological theory if selected functions can play this role. But to argue that the etiological theory is true, one must compare it to all of the best available alternatives comprehensively, and nothing like that is attempted here. I have engaged in that still ongoing debate elsewhere.
} 
Second, and this will be true on any account of normal-proper function, the analysis of a normal system will abstract from pathology. Tolstoy famously remarks that, "All happy families are alike; each unhappy family is unhappy in its own way." 29 Along similar lines, normally functioning systems (of a type) are much more alike than abnormal ones are. There are different ways to be normal (as noted in a moment), and there are commonalities among individuals with the same syndrome. But there is no limit on pathological variation other than the laws of nature and death, whereas normal functioning is more highly constrained.

Rarely will any individual be normal in every respect. So, one might ask, how is the idealization to normal-proper functioning useful if the actual functioning of individuals is not being accurately represented? In part, the answer is that successful predictions can often be made by assuming that an individual is normal in the absence of explicit evidence to the contrary. ${ }^{30}$ In practice, this might be the best that we can manage, since predictions concerning physiological outcomes in particular individuals are based on limited information about those individuals. But what is known about the actual capacities (minimal functions) of an individual's system need not be lost. If such knowledge is available, it can be taken into account. No predictive power need be lost in producing descriptions of how systems of a type function when they are functioning properly, and much is gained if individuals are mostly normal most of the time.

Thus, given the first and second points, the description of the normal system satisfies the basic task of providing for some generalization, as discussed in the previous section. The third point, however, is that a species' design is not completely universal for a species, and it is a virtue of selected function ascriptions that they do not overgeneralize. ${ }^{31}$ Even if $51 \%$ of human gonads are sperm producers (because $51 \%$ of humans are male), ova production is normal for ovaries if there was selection for ova production by ovaries. In addition to sexual dimorphism, selected functions accommodate different developmental stages (infants and adults, tadpoles and toads, caterpillars and butterflies) since there can be selection for the performance of different activities at different stages of life. And, to the extent that different populations have different selection histories in different environments, they can have population-specific norms of functioning as well. For example, different types of fat deposits, skin pigmentations and digestive enzymes in humans can be selected in response to different climates, food sources and cuisines. Also, if two strategies are preserved in a population owing to frequency-dependent contributions to fitness, the notion of selected function accommodates this. For example, within a strain of bacteria, antibiotic resistance gained by 'altruistic' production of a protective element that also protects nearby cells or by 'cheater' reliance on its production by neighbors can both be normal. There is thus

\footnotetext{
29 This is the opening line in Anna Karenina.

30 This is Dennett (1989) insight.

31 This relates to what is known as the reference-class problem for bio-statistical theories of normal function, mentioned again later.
} 
generalization without overgeneralization in describing a species design by describing what is normal, on the etiological theory. ${ }^{32}$

Moreover, physiologists need not let go of what Dennett (1989) calls 'the design stance', nor drop down to the physical stance, when they explain or predict pathology, although they can use the physical stance too. Rather, and this is the fourth point, the design stance helps to explain abnormal as well as normal functioning. For instance, suppose we know that enzyme $e$ is normally required for converting GLA to its elongated product DGLA, and that DGLA is normally required for process $P$ in immunity. Suppose that we also know that a person has an impaired capacity to produce enzyme $e$. Then, we can predict and explain the person's deficit with respect to process $\mathrm{P}$ on the basis of the known lack of $e$ and our understanding of its normal role in P. Working backwards, if we learn that an individual is impaired with respect to process $\mathrm{P}$, understanding how $\mathrm{P}$ is normally achieved will suggest (among other possibilities) the hypothesis that the individual lacks $e$, and we can test for this.

Using a notion of minimal function on its own, physiologists could in principle describe all of the actual functioning of organic systems. But in practice there are severe limits. Each complex creature would need a sui generis functional analysis, which would require constant updating as the creature aged, became sick or disabled, recovered and so on. It is not even clear that it is in practice feasible to describe all of the ways that a human could malfunction. Describing normal systems and dysfunction as a deviation from what is normal helps to alleviate this problem; it makes the description of malfunction more efficient. The description of the normal system provides a framework on which descriptions of dysfunction can be hung. To describe the functional organization that results from a broken thumb, one need not re-describe the whole system. In fact, even when dysfunction leads to widespread disruption, the resulting disorganization can often be described as specifiable tendencies to deviate from what is normal. ${ }^{33}$ Something similar is true for idiosyncratic variations that improve on normal functioning or maladaptive or adaptive consequences of sudden or local environmental change. This all requires a stable notion of normal-proper function that does not shift with researcher interests.

Stability is important in knowledge acquisition in another way. Call this the fifth point. Experimentalists often try to isolate components of complex systems as much as possible and study them in a small number of individuals in highly controlled and often artificial environments; however, in doing so, they aim to contribute to the collective enterprise of explaining how overall systems of a type normally function. For example, they aim to explain normal human immunity or normal human vision. By engaging in such research, researchers try to contribute to the "composite portrait" of a species (to borrow a phrase). ${ }^{34}$ Such experimental studies are for this reason

\footnotetext{
32 There's room for individual standards of normal functioning if ontogenetic selection is involved or if an ontogenetic process has the selected function to calibrate a system's operation in different ways depending on an individual's circumstances.

33 The subtractivity assumption is that, when impairment does occur, it does not so greatly alter other parts of the system as would in effect create a radically different system. This will not always hold true but it is a useful starting assumption.

${ }^{34}$ From Boorse, quoted in the penultimate section of this paper.
} 
explicitly reported in the titles, abstracts and main texts of research papers as studies on normal or abnormal subjects, as the case may be. This vast scientific endeavor would be thrown into disarray if the function-dysfunction and accident-function distinctions were relativized to a researcher's idiosyncratic explanatory aims. Physiology needs a stable notion of normal-proper function-one that can be used in the same way by different researchers in different laboratories and countries over the generations and around the world. Again, the notion of selected function satisfies this desideratum.

This stable notion underwrites the fruitful, common and well-recognized biological practice of learning how systems work from observing what happens when they break down in various ways. It underwrites the practice of learning about normal dependencies among capacities of components by studying how some capacities dissociate from others when functional impairment occurs. It is a stable function-dysfunction distinction that makes this possible. Those who think that normal-proper functions are modified Cummins functions can endorse this too, but the modifications will need to stabilize the notion and not leave it drifting in the breeze of shifting explanatory aims.

The sixth, last and perhaps most important point is that physiologists describe organized complexity by describing normal-proper functions. Complexity is heterogeneity - that is, diverse components doing diverse things. Organized complexity involves the finely tuned collaboration that results from the co-adaptation of the diverse activities of diverse components. We often attend to the adaptation of a creature to its external environment, outside its scales, shell or skin. But the collection of adaptations that constitute an organism is intimately coadapted. Though there are significant exceptions, at least to a first approximation, each component in a system is in general adapted to the other components of a system as well as to the outer environment. ${ }^{35}$ In describing the properly functioning system, construed as the system in which each component that was selected to do something is able to do what it was selected to do, physiologists describe how components have been co-adapted to collaborate in response to changing circumstances in the inner and outer environment, indexed to the environment in which the relevant lineage evolved.

There is thus an explanatory coherence to the functional analysis of the normal system. Obviously, we do not explain how glucose is removed from the bloodstream in a functional analysis that reports that glucose is removed from the bloodstream through the production of insulin (normal) and also reports that the pancreas produces no insulin (abnormal). Of course, a multi-level componential analysis that uses a minimal notion of function can also achieve coherence by describing the actual functioning of the components of a particular individual. At a glance, minimal functions seem to suffice for the physiologists for this very reason, especially given that most of the day-today work in physiology is on specific components in a small number of individuals; however, as a collective, physiologists aim to achieve explanatory coherence while explaining how the many operate, not just the few.

The description of the normal system as the system that functions 'as designed' is thus not merely a generalization but a useful generalization in ways that surpass mere statistical generalization.

35 This ignores segregation distorter genes, for example, but the main line of argument holds. 


\section{Is it idealization?}

Let me try to forestall a few objections. To begin, the idea that physiologists and neurophysiologists describe normal-proper functioning, construed in terms of the etiological theory of functions, does not commit biologists to unreasonable adaptationism. Nor does it commit them to thinking that every component has a selected function, that components which have selected functions were optimally designed for them, or that selection as opposed to drift or architectural or other constraints are responsible for preserving components in a population. Selection operates within constraints, alongside drift, and can result in less than optimal designs. There can be and are also features for which there was no selection. This is all perfectly compatible with what has here been said.

Nor am I denying that physiologists describe the adaptive and, for that matter, the maladaptive physiological consequences of features that are hard to change due to developmental or architectural constraints. ${ }^{36}$ These will be widespread minimal functions and I admit that biologists speak of minimal functions (though not always by that name). Physiology is not a one- or two-concept science, and describing normalproper function(ing) does not exhaust the labor involved.

Someone might dismiss the role of normal-proper function ascriptions as mere idealization. But, if the role is of scientific importance, as argued here, then it ought not to be dismissed whether it is one of idealization or not. We all want to be healthy and much of the funding for research in physiology is motivated by clinical or curative goals. And so perhaps the contrary thought is that the role of the idealization is merely a social, clinical or ethical one. The thought might be that 'pure physiology', once separated from social, clinical and ethical concerns, has no use for talk of normalproper function, but I hope that I have already said enough to dispense with such doubts.

Actually, it is a good question whether the description of a properly functioning system is an idealization. Scientific idealizations are often understood to involve falsification or simplification. Selected functions are not as-if properties, nor are they ascribed to fictional components. Many real components of organic systems really have selected functions. So a description of a system's proper functioning is not a falsification. (Unless, that is, it is a false description of a system's proper functioning or is wrongly offered as a description of a system's actual functioning when the system is not actually functioning properly.) And the properly functioning system is not as a rule simpler than a malfunctioning version of the same type of system. In fact, a degree of proper functioning is required if the complexity of the system is to be sustained. The multi-level functional analysis of a system's proper functioning can also be just as detailed as one could possibly want, at least to the point where an entirely physical description takes over.

Still, the goal of giving composite portraits of properly functioning systems of a type or kind is plainly much more manageable than that of giving a multitude of individual analyses of how individual instances actually function. And there is some

36 I here address a concern raised by Godfrey-Smith (1993). 
abstraction from detail involved with respect to variations among instances. There is abstraction from dysfunction and idiosyncratic variation and so on.

I have no commitment to any particular account of scientific idealization, and so I will not try to settle whether this is really a case of scientific idealization or not, but it is anyway not a case of mere idealization in any way that undermines its scientific significance.

Finally, this paper does not address the concern that ascriptions of selected function are too onerous in an epistemic sense for physiologists and neurophysiologists. This is a concern raised by Kitcher (1993), whose view is discussed in the next section. It is a concern that I respect and have discussed elsewhere. ${ }^{37}$ But I will here add that the key question is not if such function ascriptions are onerous but if they are more onerous than they need to be for the theoretical purposes served. Too often, the question has been discussed without an adequate understanding of the theoretical purposes served. Furthermore, it is worth remembering that physiologists and neurophysiologists can preserve epistemic caution in ascribing selected functions, alongside ascriptions of widespread minimal functions. Function ascriptions are not all or nothing when it comes to the certitude required. Biologists can (and often do) speak of what the functions of components likely are, are thought to be and so on. There is at least a stable objective target to be described if the aim is to describe the system that is functioning 'as designed' - that is, if the aim is to describe the complex of properly functioning co-adapted components, in which each component that was selected to do something is able to do what it was selected to do.

\section{Related views}

This penultimate section looks at some related views. The fact that physiologists and neurophysiologists idealize (I shall continue to use the word) to normal functioning has certainly not gone unnoticed. But it has, to a surprising extent, managed to stay beneath the radar when the question of which notion(s) of function they use in answering Howquestions is discussed.

Sometimes it is said that the 'laws' of physiology and neurophysiology are ceterus paribus laws that apply to 'intact' systems, which are implicitly or explicitly understood to be properly functioning systems. This recognizes the background idealization to normal-proper functioning, but it has the effect of sweeping the significance of the relevant notion of function under the carpet. It places the possibility of malfunction and other sources of idiosyncratic variation alongside meteor strikes as freak interfering factors. And, by so doing, it implies that physiologists are only describing what the components of complex systems do in the usual way that all scientists do whenever they are forced to work with laws of nature that are not without exception.

Christopher Boorse has a different appreciation of the role of the notion of normalproper function, which is a lot closer to the one endorsed here. But, in his view, physiologists are making statistical generalizations, if not mere statistical generaliza-

\footnotetext{
37 See Neander (forthcoming), which argues that Kitcher's concerns about the onerous nature of selected functions relies upon an unnecessarily strong etiological notion. See also Neander (1999).
} 
tions. When physiologists describe the organisms in a species, he says, "Each detail of this composite portrait is statistically normal within the species, though the portrait may not exactly resemble any species member ... since any frog is bound to be atypical in some respect and to have suffered the ravages of injury or disease" (Boorse 1977, p. 557). Work on what are called 'biostatistical theories' continues, but these theories still have unresolved and, in my view, insoluble problems. ${ }^{38}$ Since a discussion of these problems lies beyond the scope of this paper, suffice it to say that even the proponents of such theories deny that talk of normal function merely involves standard scientific use of statistics. Boorse's account, for instance, appeals to a cybernetic notion of goaldirectedness and a (problematic, because seemingly circular) notion of a reference class of uniform functional design within a species. Of course, physiologists and neurophysiologists can and do make statistical generalizations; however, mere statistical generalization is not what is distinctive about their descriptions of normal systems.

Two other views worth a closer look are those of Godfrey-Smith and Kitcher, both of whom have pluralist views, on which an etiological notion of function is used to answer Why-questions and something closer to Cummins' original notion of function is used to answer How-questions.

Godfrey-Smith notes that a function-dysfunction distinction can be accommodated within a Cummins-style approach, as well as within Wright's. He does not elaborate much on this but adds, "If a token of a component of a system is not able to do what other tokens do, that plays a distinguished role in the explanation of the capacities of the broader system, then that token component is malfunctioning" (1993, p. 200). His use of the type-token distinction makes space for token malfunction. ${ }^{39}$ However, Godfrey-Smith also recommends that Cummins functions be allowed to roam free, with the relevant $\left(Z^{*}\right)$ complex capacity to be determined by researcher interests. And he does so even though, as he remarks, some such functions will be functions in virtue of their being contributions to pathological processes (the malignant growth of tumors and so on) when researchers are interested in explaining these pathological processes. So, while this way of interpreting or modifying Cummins' original proposal provides for the possibility of some token 'malfunction', it will turn the standard functiondysfunction distinction on its head in some explanatory contexts.

However, instead of allowing modified Cummins functions to roam free, we could rein them in, in the way suggested by Kitcher. Like Godfrey-Smith, Kitcher (1993) holds a pluralist view, according to which both an etiological notion and a more Cummins-like notion of function have distinct explanatory roles. Again, he thinks that the latter notion is used in explaining how systems operate, unless evolutionary

\footnotetext{
38 Here are three starting problems. (1) There is no conceptual incoherence in the idea that dysfunction can become typical, at least for a time, and thus the relevant time period for the statistics needs specifying in a non-circular way. (2) Some normal functions are rarely performed. E.g., only a minute proportion of sperm fertilize ova. (3) A statistical theory requires the specification of a reference-class within which normal functioning is typical, but an adequate non-circular specification of relevant reference classes proves elusive. For discussion (both for and against statistical accounts), see Boorse (1977, 2002), Garson and Piccinini (2013), Kingma (2007, 2010), Kraemer (2013), Neander (1991a, 2002), and Schwartz (2007).

39 Cummins (1996) endorses this when he says that, on all theories of functions, functions belong to tokens in virtue of their being of a type. The implication is that Cummins intends his (1975) proposal to be read in this way.
} 
questions are explicitly moved to the forefront. But Kitcher sees more unity of conception in the two notions. ${ }^{40}$ On his view, the main difference between them lies in whether the link to selection is more or less direct. On his view, a component has a 'strong etiological function' (as he calls it) to do Z only if the component is designed to do Z, whereas a component has a 'causal-role' function' (as he calls it) to do Z as long as it contributes Z-ing to something that its containing system is designed to do. In the second case, there need be no selection in favor of a component's function. If jackrabbits have been adapted for thriving in a desert, any contribution (accidental or not) to a jackrabbit's thriving in a desert by some component of the jackrabbit (or some feature of such a component, such as the length of its ears) will be a causal role function. So Kitcher's causal role functions are Cummins functions that contribute to what the overall system is designed to do, which in the case of organisms (as opposed to artifacts) will be to survive and reproduce under certain circumstances. His notion of a causal role function does not try to capture the function-accident distinction, but it does try to capture the function-dysfunction distinction. Components do not have causal role functions to contribute to malignancy, on Kitcher's account (unless programmed death is part of the design).

In Kitcher's view, physiologists use a notion of normal-proper function to explain how systems operate. But, in his view (as in Boorse's and Godfrey-Smith's) this is not an etiological notion of a selected function. It is a Cummins function, with the modification that the relevant $Z^{*}$ capacities of whole organisms are fixed, not by researcher interests, but by what the system as a whole is designed to do (whereas the various $\mathrm{Z}$ capacities of their components are not fixed by what, if anything, the components were designed to do).

Now it is harder to decide whether selected functions or these modified Cummins functions are to be preferred for identification with the normal-proper functions of which the physiologists speak in answering How-questions. We would need more details before their virtues could be usefully compared. Various versions of the etiological theory are described elsewhere (including those by Godfrey-Smith and Kitcher). ${ }^{41}$ With respect to causal role functions, we will need to obtain answers to the following questions, to start with. If the normal function of $x$ is to do $z$, is it necessary that any present $x$ s do $z$ ? If so, (roughly) how many-one, some, many, most? Or will the fact that some $x \mathrm{~s}$ did $z$ in the relatively recent past suffice? If so, how frequently must this have happened in the past? For example, must it recently have been typical of $x$ s that they do $z$ ? Or is the answer that $x \mathrm{~s}$ must have done $z$ often enough for selection of $x \mathrm{~s}$ for doing $z$ to have occurred? Also, how are $x \mathrm{~s}$ to be typed (as $x \mathrm{~s}$ ) for these purposes? Are they $x$ s in virtue of belonging to lineages of homologs? And in which environment must some $x$ s be able to do or have been able to do $z$ ? Is it the current environment in

\footnotetext{
40 On Kitcher's (1993) theory, having been selected to do Z suffices for being designed to do Z. Kitcher uses the tenseless expression 'is designed to do Z', but it seems to be an appeal to history. There are, however, ahistorical suggestions that one could make instead. For instance, Boorse (2002) identifies the ultimate 'goals' of a system in cybernetic terms.

41 For Godfrey-Smith's version, see Godfrey-Smith (1994). Kitcher (1993) is as far as I know Kitcher's principal discussion of functions.
} 
which the organism is found, whether or not it is the organism's 'natural habitat'? Or is it the one in which organisms of the type evolved relatively recently?

Some answers will turn modified Cummins functions into selected functions. Others will turn them into bio-statistical functions. Still others will turn them into something else. ${ }^{42}$ Each proposal has to be considered on its merits, but we first need a sufficiently developed proposal before us.

Of course, one could choose to be an instrumentalist at various points in answer to the questions listed above. The instrumentalist can deny that there are general answers to be given, and can instead maintain that the answers are determined by the pragmatics of the explanatory context on a case-by-case basis. But, for the reasons mentioned earlier, this is not a choice to be taken lightly.

I should add that selected function ascriptions are usefully refined with their role in multi-level componential analyses of complex systems in mind. ${ }^{43}$ Selected functions can be complex causal roles (for which there was selection), which can involve interactions with other components in a system as well as with various environmental features, and such functions are appropriately described in different ways depending on which level(s) of a system's operation and which aspect(s) of its operation we are interested in explaining. The appropriateness of an ascription is certainly relative to explanatory aims.

\section{Concluding remarks}

It is apparently tempting to think that Cummins-style functions and not Wright-style selected functions serve the explanatory needs of physiologists. One motivation is seemingly that selected functions lack causal efficacy in the operation of bodies and brains. It is thought to follow that how a system operates is explained by what components do and not what they were selected to do. But causal contributions qua causal contributions are not Cummins functions, which are causal contributions to complexly achieved capacities that someone is interested in explaining. Moreover, physiologists and neurophysiologists use a notion of normal-proper function, as well as a minimal notion of function (of mere activity). And normal-proper functions must lack causal efficacy whether they are selected functions or modified Cummins functions. It is argued in this paper that ascriptions of normal-proper function to the components of living systems play a significant scientific role in biological explanations of how living systems operate. It is also argued that this role may be played by ascriptions of selected function. Biologists describe normal-proper functions in explaining how bodies and brains operate because this generates useful, general descriptions of organized complexity. Easy confidence in the claim that instrumentalist Cummins-style functions as opposed to Wright-style selected functions are appropriate for answering How-questions is ill founded.

\footnotetext{
42 Nanay (2010) offers a radically different alternative, but see Neander and Rosenberg's (2012) response.

43 See, e.g., Griffiths (1993) and Neander (1995).
} 
Acknowledgements I am grateful to the anonymous referees for Synthese who persevered in forcing me to clarify the argument of this paper. Earlier versions of the paper were presented at colloquia, workshops and conferences over the past decade. I want to thank the organizers and the members of the audiences for the stimulating discussions from which I benefited on those occasions. Early versions (under various titles) were presented at the 'Functions in Action Workshop', in Heidelberg, Germany, on January 11, 2007; at the Department of Philosophy, Virginia Commonwealth University, September 28th, 2007; at the 'Form, Function and Homology Conference: Duke's 8th Annual Conference in Philosophy \& Biology and the Annual Consortium for the History and Philosophy of Biology', on May 22nd, 2009, at Duke University; and in the workshop, 'Norms and Teleological Organization', in Copenhagen, Denmark, on July 2012, which was organized by John Basl and Sune Holm. I also want to thank John Basl for pressing me to submit the paper to Synthese for this issue.

Open Access This article is distributed under the terms of the Creative Commons Attribution 4.0 International License (http://creativecommons.org/licenses/by/4.0/), which permits unrestricted use, distribution, and reproduction in any medium, provided you give appropriate credit to the original author(s) and the source, provide a link to the Creative Commons license, and indicate if changes were made.

\section{References}

Amundson, R., \& Lauder, G. (1994). Function without purpose. Biology and Philosophy, 9(4), 443-469. Bock, W. J., \& von Wahlert, G. (1965). Adaptation and the form-function complex. Evolution, 19, 269-299. Boorse, C. (1977). Health as a theoretical concept. Philosophy of Science, 44, 542-573.

Boorse, C. (2002). A rebuttal on functions. In A. Ariew, R. C. Cummins, \& M. Perlman (Eds.), Functions: New essays in the philosophy of psychology and biology. New York: Oxford University Press.

Bouchard, F. (2013). How ecosystem evolution strengthens the case for functional pluralism. In P. Huneman (Ed.), Functions: Selection and mechanisms. Dordrecht: Springer.

Brandon, R. (2013). A general case for function pluralism. In P. Huneman (Ed.), Functions: Selection and mechanisms. Dordrecht: Springer.

Craver, C. (2001). Role functions, mechanisms, and hierarchy. Philosophy of Science, 68, 53-74.

Craver, C., \& Darden, L. (2013). In search of mechanisms, discoveries across the life sciences. Chicago, IL: The University of Chicago Press.

Cummins, R. (1975). Functional analysis. The Journal of Philosophy, 72(20), 741-765.

Cummins, R. (1996). Representations, targets, and attitudes. Cambridge: MIT press.

Cummins, R., \& Roth, M. (2010). Traits have not evolved to function the way they do because of a past advantage. In F. Ayala \& R. Arp (Eds.), Contemporary debates in philosophy of biology (pp. 72-88). Oxford: Blackwell.

Davidson, D. (1987). Knowing one's own mind. Proceedings and addresses of the American philosophical association, 60(3), 441-458.

Davies, P. S. (2001). Norms of nature: Naturalism and the nature of functions. Cambridge, MA: MIT Press.

Dayal, S., et al. (2008). Tissue-specific downregulation of dimethylarginine dimethylaminohydrolase in hyperhomocysteinemia. American Journal of Physiology_Heart and Circulatory Physiology, 295(2), H816-H825.

Dennett, D. C. (1989). The intentional stance. Cambridge: MIT press.

Dretske, F. (1988). Explaining behavior: Reasons in a world of causes. Cambridge, MA: MIT Press.

Garson, J. (2012). Function, selection and construction in the brain. Synthese, 189(3), 451-481.

Garson, J. (2013). The functional sense of mechanism. Philosophy of Science, 80, 317-333.

Garson, J., \& Piccinini, G. (2013). Functions must be performed at appropriate rates in appropriate situations. The British Journal for the Philosophy of Science. doi:10.1093/bjps/axs041.

Godfrey-Smith, P. (1993). Functions: Consensus without unity. Pacific Philosophical Quarterly, 74(3), 196-208.

Godfrey-Smith, P. (1994). A modern history theory of functions. Noûs, 28(3), 344-362.

Griffiths, P. E. (1993). Functional analysis and proper functions. The British Journal for the Philosophy of Science, 44(3), 409-422.

Griffiths, P. E. (2006). Function, homology, and character individuation. Philosophy of Science, 73(1), 1-25.

Hardcastle, V. G. (1999). Understanding functions: A pragmatic approach. In V. Hardcastle (Ed.), Where biology meets psychology, philosophical essays. Cambridge, MA: MIT Press. 
Hempel, C. G. (1965). Aspects of scientific explanation; and other essays in the philosophy of science. New York: Free Press.

Huneman, P. (2013). Introduction. In P. Huneman (Ed.), Functions: Selection and mechanisms. Dordrecht: Springer.

Kim, J. (2000). Mind in a physical world: An essay on the mind-body problem and mental causation. Cambridge: MIT press.

Kim, J. (2010). Philosophy of Mind. Westview Press.

Kingma, E. (2007). What is it to be healthy? Analysis, 67(2), 128-133.

Kingma, E. (2010). Paracetamol, poison, and polio: Why Boorse's account of function fails to distinguish health and disease. British Journal for the Philosophy of Science, 61(2), 241-264.

Kitcher, P. (1993). Function and design. Midwest Studies in Philosophy, 18(1), 379-397.

Kraemer, D. M. (2013). Statistical theories of functions and the problem of epidemic disease. Biology and Philosophy, 28(3), 423-438.

Kraemer, D. M. (2014). Normativity from an organizational perspective. Biological Theory, 9(3), $253-257$.

Laviola, L., et al. (2008). Abnormalities of IGF-I signaling in the pathogenesis of diseases of the bone, brain, and fetoplacental unit in humans. American Journal of Physiology Endocrinology and Metabolism, 295(5), E991-E999.

Laviano, A., et al. (2008). Neural control of the anorexia-cachexia syndrome. American Journal of Physiology-Endocrinology and Metabolism, 295(5), E1000-E1008.

Mayr, E. (1961). Cause and effect in biology: Kinds of causes, predictability, and teleology are viewed by a practicing biologist. Science, 134(3489), 1501-1506.

Mayr, E. (1992). The idea of teleology. Journal of the History of Ideas, 53(1), 117-135.

Millikan, R. G. (1989a). In defense of proper functions. Philosophy of Science, 56(2), 288-302.

Millikan, R. G. (1989b). An ambiguity in the notion "function". Biology and Philosophy, 4(2), 172-176.

Millikan, R. G. (2002). Biofunctions: Two paradigms. In A. Ariew, R. C. Cummins, \& M. Perlman (Eds.), Functions: New essays in the philosophy of psychology and biology. New York: Oxford University Press.

Nanay, B. (2010). A modal theory of function. The Journal of Philosophy, 107(8), 412-431.

Neander, K. (1983). Abnormal psychobiology. Thesis dissertation, La Trobe.

Neander, K. (1991a). Functions as selected effects: The conceptual analyst's defense. Philosophy of Science, 58(2), 168-184.

Neander, K. (1991b). The teleological notion of 'function'. Australasian Journal of Philosophy, 69(4), 454-468.

Neander, K. (1995). Misrepresenting \& malfunctioning. Philosophical Studies, 79(2), 109-141.

Neander, K. (1996). Swampman meets swampcow. Mind and Language, 11(1), 118-129.

Neander, K. (1999). Fitness and the fate of unicorns. In V. Gray Hardcastle (Ed.), Where Biology Meets Psychology. MIT Press.

Neander, K. (2002). Types of traits: The importance of functional homologues. In A. Ariew, R. Cummins, \& M. Perlman (Eds.), Functions: New essays in the philosophy of biology and psychology (pp. 390-415). New York: Oxford University Press.

Neander, K. (2011). Teleological theories of mental content. In Stanford encyclopedia of philosophy (online).

Neander, K. (forthcoming). Kitcher's two design stances. In M. Crouch \& J. Pfeifer (Eds.), Kitcher and his critics. Oxford: Oxford University Press.

Neander, K., \& Rosenberg, A. (2012). Solving the circularity problem for functions: A response to Nanay. Journal of Philosophy, 109(10), 613-622.

Rey, G. (1997). Contemporary philosophy of mind: A contentiously classical approach. Oxford: Blackwell.

Salmon, W. C. (1993). The value of scientific understanding. Philosophia, 51(1), 9-19.

Sober, E. (1994). The nature of selection: Evolutionary theory in philosophical focus. University of Chicago Press.

Sober, E. (1995). Natural selection and distributive explanation: A reply to Neander. British Journal for the Philosophy of Science, 46(3), 384-397.

Schwartz, P. H. (2007). Defining dysfunction: Natural selection, design, and drawing a line. Philosophy of Science, 74(3), 364-385.

Wright, L. (1973). Functions. The Philosophical Review, 82, 139-168.

Wright, L. (1976). Teleological explanations. Berkeley, CA: University of California Press. 